\title{
Single-flavor CSL phase in compact stars
}

\author{
David Blaschke,, Fredrik Sandinn ${ }^{*,+\sharp}$, Thomas Klähn ${ }^{\S}$ and \\ Jens Berdermann \\ *Instytut Fizyki Teoretycznej, Uniwersytet Wroctawski, 50-204 Wrockaw, Poland \\ ${ }^{\dagger}$ Bogoliubov Laboratory for Theoretical Physics, JINR, 141980 Dubna, Russia \\ ** Département d'Astrophysique, de Géophysique et d'Océanographie, Université de Liège, \\ B5a, Sart-Tilman, B-4000 Liège, Belgium \\ ${ }^{\ddagger}$ Division of Physics, Luleå University of Technology, SE-97187 Luleå, Sweden \\ $\S$ Theory Division, Argonne National Laboratory, Argonne IL, USA \\ 'DESY Zeuthen, Platanenallee 6, D-15738 Berlin, Germany \\ E-mail: Blaschke@ift.uni.wroc.pl,JF.Sandin@ulg.ac.be,Thomas.Klaehn@googlemail.com, \\ Jens.Berdermann@googlemail.com
}

\begin{abstract}
We suggest a scenario where the three light quark flavors are sequentially deconfined under increasing pressure in cold asymmetric nuclear matter as, e.g., in neutron stars. The basis for our analysis is a chiral quark matter model of Nambu-Jona-Lasinio (NJL) type with diquark pairing in the spin-1 single flavor (CSL), spin-0 two flavor (2SC) and three flavor (CFL) channels. We find that nucleon dissociation sets in at about the saturation density, $n_{0}$, when the down-quark Fermi sea is populated (d-quark dripline) due to the flavor asymmetry induced by $\beta$-equilibrium and charge neutrality. At about $3 n_{0} \mathrm{u}$-quarks appear and a two-flavor color superconducting (2SC) phase is formed. The s-quark Fermi sea is populated only at still higher baryon density, when the quark chemical potential is of the order of the dynamically generated strange quark mass. We construct two different hybrid equations of state (EoS) using the Dirac-Brueckner Hartree-Fock (DBHF) approach and the EoS by Shen et al. in the nuclear matter sector. The corresponding hybrid star sequences have maximum masses of, respectively, 2.1 and $2.0 \mathrm{M}_{\odot}$. Two- and three-flavor quark-matter phases exist only in gravitationally unstable hybrid star solutions in the DBHF case, while the Shen-based EOS produce stable configurations with a 2SC phase component in the core of massive stars. Nucleon dissociation due to d-quark drip at the crust-core boundary fulfills basic criteria for a deep crustal heating process which is required to explain superbusts as well as cooling of X-ray transients.
\end{abstract}

Keywords: Quark Gluon Plasma, Nonperturbative Models, Color Superconductivity PACS: $12.38 . \mathrm{Mh}, 11.10 . \mathrm{St}, 12.38 . \mathrm{Lg}$

\section{INTRODUCTION}

The phenomenology of compact stars is intimately connected to the EoS of matter at densities well beyond the nuclear saturation density, $n_{0}=0.16 \mathrm{fm}^{-3}$. Compact stars are therefore natural laboratories for the exploration of baryonic matter under extreme conditions, complementary to those created in terrestrial experiments with atomic nuclei and heavy-ion collisions. Recent results derived from observations of compact stars provide serious constraints on the nuclear EoS, see [1] and references therein. A stiff EoS at high density is needed to explain the high compact-star masses, $M \sim 2.0 \mathrm{M}_{\odot}$, reported for some low-mass X-ray binaries (LMXBs), e.g., $4 \mathrm{U} 1636-536$ [2], and the large radius, $R>12 \mathrm{~km}$, of the isolated neutron star

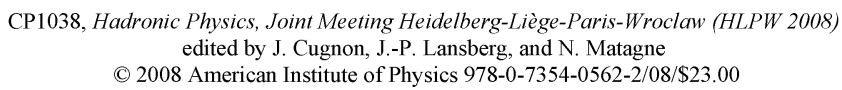


RX J1856.5-3754 (shorthand: RX J1856) [3]. Another example is EXO 0748-676, an LMXB for which the compact-star mass and radius have been constrained to $M \geq 2.10 \pm 0.28 \mathrm{M}_{\odot}$ and $R \geq 13.8 \pm 0.18 \mathrm{~km} \mathrm{[4].} \mathrm{However,} \mathrm{the} \mathrm{status} \mathrm{of} \mathrm{the} \mathrm{results}$ for the latter object is unclear, because the gravitational redshift $z=0.35$ observed in the X-ray burst spectra [5] has not been confirmed, despite numerous attempts. While compact-star phenomenology apparently points towards a stiff EoS at high density, heavy-ion collision data for kaon production [6] and elliptic flow [7] set an upper limit on the stiffness of the EoS [1].

A key question regarding the structure of matter at high density is whether a phase transition to quark matter occurs inside compact stars, and whether it is accompanied by unambiguous observable signatures. It has been argued that the observation of a compact star with high mass and large radius, likewise reported for EXO 0748-676, would be incompatible with a quark core [4], because the softening of the EoS due to quark deconfinement lowers the maximum mass and the radius in comparison to the nuclear matter case. However, Alford et al. [8] have demonstrated with a few counter examples that quark matter cannot be excluded by this argument. In particular, for a recently developed hybrid star EoS [9], based on the DBHF approach in the nuclear sector and a three-flavor chiral quark model [10], stable hybrid stars in the mass range from $1.2 \mathrm{M}_{\odot}$ up to $2.1 \mathrm{M}_{\odot}$ have been obtained, in accordance with modern mass-radius constraints, see also [11]. In that model, a sufficiently low critical density for quark deconfinement was achieved with a strong diquark coupling, while a sufficient stiffness for a high maximum mass of the compact star sequence was obtained with a repulsive vector meanfield in the quark matter sector. The corresponding hybrid EoS for symmetric matter was shown to fulfill the constraints from elliptic flow data in heavy-ion collisions. In the present work we want to discuss a new scenario of quark deconfinement, which could play an important role in asymmetric matter, in particular for the phenomenology of compact stars.

Chiral quark models of the NJL type with dynamical chiral symmetry breaking have the property that the restoration of this symmetry (and the related quark deconfinement) at zero temperature is flavor specific. When solving the gap and charge-neutrality equations selfconsistently one finds that the chiral symmetry restoration for a given flavor occurs when the chemical potential of that flavor reaches a critical value that is approximately equal to the dynamically generated quark mass, $\mu_{f}=\mu_{c} \approx m_{f}$, where $f=u, d, s$. In asymmetric matter the quark chemical potentials are different. Consequently, the NJL model behavior suggests that the critical density of deconfinement is flavor dependent, see Fig. 1. In this approach the down quark flavor is the first to drip out of nucleons as the density increases, followed by the up quark flavor and eventually also by strange quarks. This behavior is absent in simple and commonly applied thermodynamic bag model equations of state since they are essentially flavor blind.

Under the $\beta$-equilibrium condition in compact stars the chemical potentials of quarks and electrons are related by $\mu_{d}=\mu_{s}$ and $\mu_{d}=\mu_{u}+\mu_{e}$. The mass difference between the strange and the light quark flavors $m_{s} \gg m_{u}, m_{d}$ has two consequences: (1) the down and strange quark densities are different, so charge neutrality requires a finite electron density and, consequently, (2) $\mu_{d}>\mu_{u}$. When increasing the 

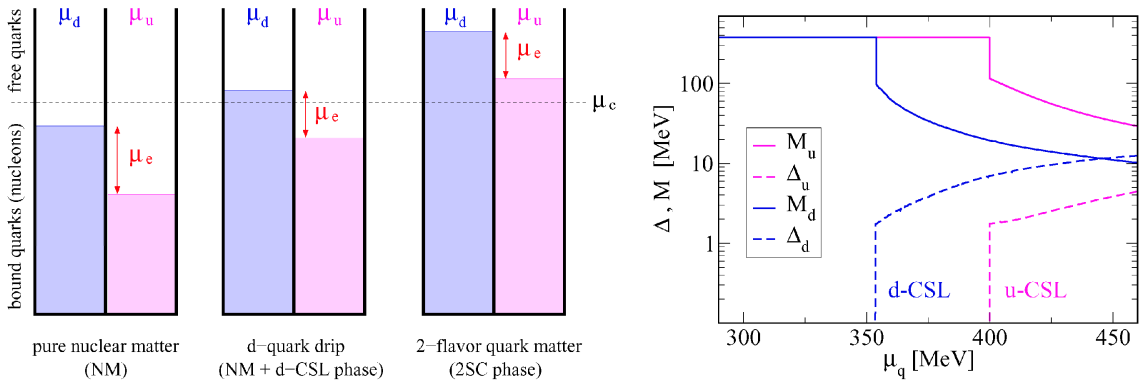

FIGURE 1. Left panel: Chemical potentials of up and down quarks (strange quark sector not shown). With increasing quark chemical potential $\mu_{q}=\left(\mu_{u}+\mu_{d}\right) / 2$ in isospin asymmetric matter the quark flavors pass sequentially the threshold $\left(\mu_{c}\right)$ for chiral symmetry restoration (deconfinement), which entails nucleon dissociation. Right panel: Solution of the NJL gap equations under isospin asymmetry.

baryochemical potential, the d-quark chemical potential is therefore the first to reach the critical value $\mu_{c}$ where the chiral symmetry gets (approximately) restored in a first-order transition and a finite density of d-quarks appears. Due to the finite value of $\mu_{e}$, the u-quark chemical potential is still below $\mu_{c}$ while the s-quark density is zero due to the high s-quark mass. A single-flavor d-quark phase therefore forms in co-existence with the positively charged nuclear-matter medium.

Why has this interesting scenario been left unnoticed? On the one hand, bag models, which are commonly used to describe quark matter in compact star interiors cannot address sequential deconfinement. On the other hand, the singleflavor d-quark phase is negatively charged and cannot be neutralized in a purely leptonic background. This was a reason to disregard it in dynamical approaches like the NJL models. In the following we discuss the single-flavor phase for the first time under the natural assumption that the neutralizing background is nuclear matter. Since nucleons are bound states of quarks, the physical context in which such a mixed phase of nucleons and free d-quarks occurs is that of the dissociation of nucleonic bound states of quarks (Mott effect).

\section{PHASE TRANSITION TO QUARK MATTER: NUCLEON DISSOCIATION}

The task to develop a unified description of the phase transition from nuclear matter to quark matter on the quark level, as a dissociation of three-quark bound states into their constituents in the spirit of a Mott transition has not been solved yet. Only some aspects of such a description have been revealed within a nonrelativistic potential model $[12,13]$ and within the NJL model [14]. We will consider a chemical equilibrium of the type $n+n \leftrightarrow p+3 d$, which results in a mixed phase of nucleons and down quarks once the d-quark chemical potential exceeds the critical value. This scenario is analogous to the dissociation of nuclear clusters 

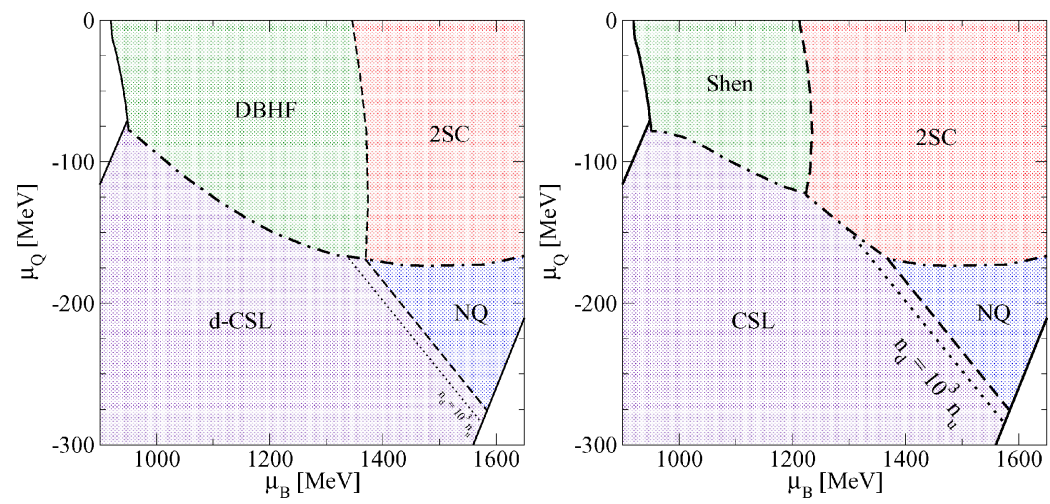

FIGURE 2. Phase diagram in the plane of baryon and charge chemical potential. The dashdotted line denote the border between oppositely charged phases. The nuclear matter EoS is DBHF (left panel) and Shen et al. (right panel).

in the crust of neutron stars (neutron dripline) and the effect may therefore be called the $d$-quark dripline. We approximate the quark and nucleon components as subphases described by separate models. For the nuclear matter subphase we use two alternatives: (1) the DBHF approach $[15,16,17,18,19]$ with the relativistic Bonn A potential, where the nucleon selfenergies are based on a $\mathrm{T}$ matrix obtained from the Bethe-Salpeter equation in the ladder approximation and (2) the EoS by Shen et al. [20], which is based on a relativistic mean field theory and includes the contribution of heavy nuclei, described within the Thomas-Fermi approximation. The quark matter phase is described within a three-flavor NJLtype model including diquark pairing channels [10, 21, 22, 23]. The path-integral representation of the partition function is given by

$$
\begin{gathered}
Z(T, \hat{\mu})=\int \mathcal{D} \bar{q} \mathcal{D} q \exp \left\{\int_{0}^{\beta} d \tau \int d^{3} x\left[\bar{q}\left(i \not \partial-\hat{m}+\hat{\mu} \gamma^{0}\right) q+\mathcal{L}_{\text {int }}\right]\right\}, \\
\mathcal{L}_{\text {int }}=G_{S}\left\{\sum_{a=0}^{8}\left[\left(\bar{q} \tau_{a} q\right)^{2}+\left(\bar{q} i \gamma_{5} \tau_{a} q\right)^{2}\right]+\eta_{D 0} \sum_{A=2,5,7} j_{D 0, A}^{\dagger} j_{D 0, A}+\eta_{D 1} j_{D 1}^{\dagger} j_{D 1}\right\},
\end{gathered}
$$

where $\hat{\mu}=\frac{1}{3} \mu_{B}+\operatorname{diag}_{f}\left(\frac{2}{3},-\frac{1}{3},-\frac{1}{3}\right) \mu_{Q}+\lambda_{3} \mu_{3}+\lambda_{8} \mu_{8}$ is the diagonal quark chemical potential matrix and $\hat{m}=\operatorname{diag}_{f}\left(m_{u}, m_{d}, m_{s}\right)$ is the current-quark mass matrix. For $a=0, \tau_{0}=(2 / 3)^{1 / 2} \mathbf{1}_{f}$, otherwise $\tau_{a}$ and $\lambda_{a}$ are Gell-Mann matrices acting in flavor and color spaces, respectively. $C=i \gamma^{2} \gamma^{0}$ is the charge conjugation operator and $\bar{q}=q^{\dagger} \gamma^{0}$. The scalar quark-antiquark current-current interaction is given explicitly and has coupling strength $G_{S}$. The 3-momentum cutoff, $\Lambda$, is fixed by low-energy QCD phenomenology (see table I of [24]). The spin-0 and spin-1 diquark currents are $j_{D 0, A}=q^{T} i C \gamma_{5} \tau_{A} \lambda_{A} q$ and $j_{D 1}=q^{T} i C\left(\gamma_{1} \lambda_{7}+\gamma_{2} \lambda_{5}+\gamma_{3} \lambda_{2}\right) q$. While the relative coupling strengths $\eta_{D 0}$ and $\eta_{D 1}$ are essentially free parameters, 

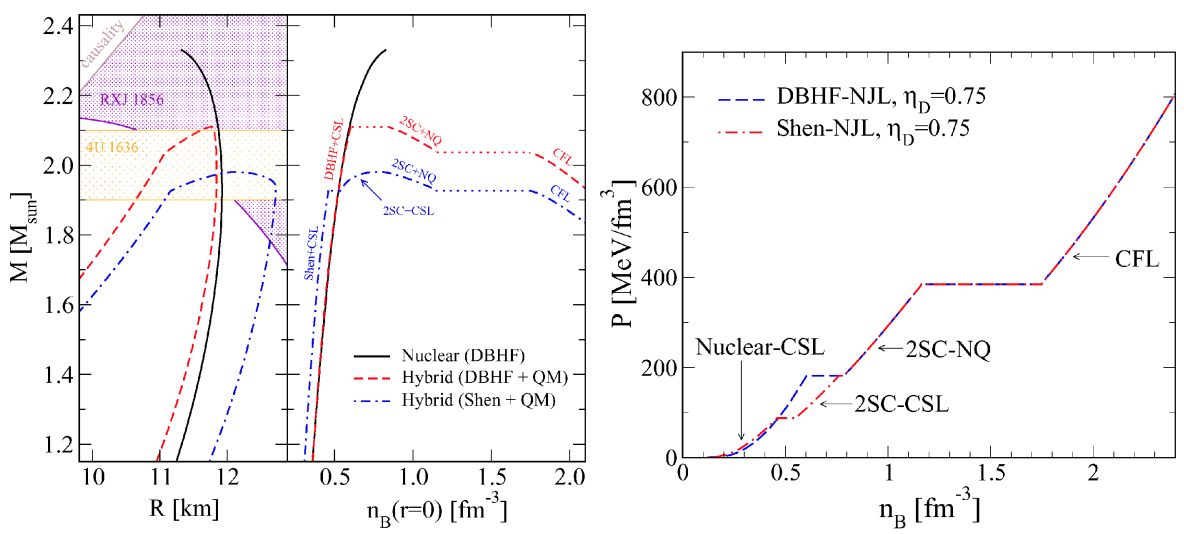

FIGURE 3. Left panel: Compact star sequences. The phase structure of the core changes with increasing density, as indicated in the figure. Constraints on the mass come from 4U 1636 [2] and on the mass-radius relation from RX J1856 [3]. Right panel: Hybrid equations of state used in the calculation of the compact star sequences.

we restrict the discussion to the Fierz values, $\eta_{D 0}=3 / 4$ and $\eta_{D 1}=3 / 8$, see [25]. Color superconducting phases in QCD with one flavor have first been discussed in Refs. [26, 27, 28], where also the special role of the spin-1 color-spin locking phase has been pointed out that here all quarks participate in the pairing with a gap of the order of $1 \mathrm{MeV}$ or even below. This feature of the CSL phase is robust, as was demonstrated for our above isotropic ansatz for the spin-1 diquark current introduced in [29], and for its generalizations to the nonlocal case [30] and to a selfconsistent Dyson-Schwinger approach [31].

The gaps and the renormalized masses are determined by minimization of the mean-field thermodynamic potential, under the constraints of charge neutrality and $\beta$-equilibrium. For further details, see [10, 21, 22, 23]. In Fig. 2 we plot the thermodynamically favored phase in the plane of baryon and charge chemical potentials.

The hybrid EoS corresponds to the dash-dotted lines in Fig. 2 and are constructed such that the mixture of nuclear matter, quark matter and leptons is charge neutral. Using the hybrid EoS we calculate the corresponding compact star sequences by solving the Oppenheimer-Volkoff equations for hydrostatic equilibrium. The hybrid-star sequences fulfill all modern constraints on the mass-radius relationship, see Fig. 3. For the DBHF hybrid EoS all stars with DBHF+CSL matter in the core are stable equilibrium solutions, while the appearance of u-quarks and the associated formation of a $2 \mathrm{SC}$ subphase renders the sequence unstable. The situation is somewhat different for the Shen hybrid EoS, because in addition to Shen+CSL stars there are stable solutions with 2SC+CSL matter in the core. In both cases configurations with strange quarks in the core are unstable.

The hybrid star sequences 'masquerade' as neutron stars [32], because the mechanical properties are similar to those of nuclear matter stars and the transition 
from nuclear matter to the mixed phase is associated with a relatively small discontinuity in the density. Unmasking neutron star interiors might therefore require observables based on transport properties, which could be strongly modified in presence of color superconductivity. It has been suggested to base such tests of the structure of matter at high density on an analysis of the cooling behavior $[33,34,35,36]$ or the stability of rapidly spinning stars against r-modes [37, 38]. It has turned out that these phenomena are sensitive to the details of color superconductivity in quark matter.

\section{BULK VISCOSITY AND URCA EMISSIVITY OF THE SINGLE-FLAVOR CSL PHASE}

According to [39] rotating compact stars would be unstable against r-modes in the absence of viscosity [40]. Constraints on the composition of compact-star interiors can therefore be obtained from observations of millisecond pulsars [37, 38]. In such investigations the bulk viscosity is a key quantity. We therefore consider some relevant aspects here, starting with the two-flavor color superconducting phases following the approach described in Ref. [41].

The temperature-dependent bulk viscosity for the 2SC-CSL phase has been calculated self-consistently in [42] and is based on the flavor-changing weak processes of electron capture and $\beta$ decay

$$
u+e^{-} \rightarrow d+\nu_{e} \quad, \quad d \rightarrow u+e^{-}+\bar{\nu}_{e} .
$$

It has been shown that the bulk viscosity is related to the direct URCA emissivity, which for normal quark matter was first calculated by Iwamoto [43] and can be expressed as

$$
\varepsilon_{0} \simeq \frac{914 \pi}{1680} G_{F}^{2} \mu_{e} \mu_{u} \mu_{d} T^{6} \theta_{u e}^{2}
$$

Here $G_{F}$ is the weak coupling constant and $\theta_{u e}$ is the angle between the upquark and electron momenta, which is obtained from momentum conservation in the matrix element, see Fig. 4. The triangle of momentum conservation holds for the late cooling stage, when the temperature is below $1 \mathrm{MeV}$ and neutrinos are untrapped. Trigonometric relations are used to find an analytical expression for momentum conservation. To lowest order in $\theta_{d e}$ the result is

$$
p_{F, d}-p_{F, u}-p_{F, e} \simeq-\frac{1}{2} p_{F, e} \theta_{d e}^{2} .
$$

For small angles $\theta_{d e} \simeq \theta_{u e}$, so it is possible to obtain an expression for the matrix element of the direct URCA process. Following Iwamoto [43] one has to account either for quark-quark interactions to lowest order in the strong coupling constant, $\alpha_{s}$, (6) or the effect of finite masses (7):

$$
\mu_{i}=p_{F, i}\left(1+\frac{2}{3 \pi} \alpha_{s}\right), \quad i=u, d
$$



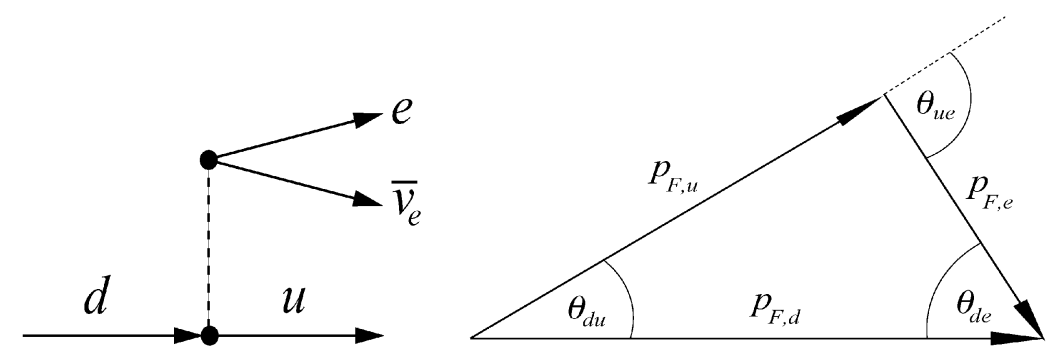

FIGURE 4. Direct Urca process in quark matter (left) and triangle of momentum conservation for it (right).

$$
\mu_{i} \simeq p_{F, i}\left[1+\frac{1}{2}\left(\frac{m_{i}}{p_{F, i}}\right)^{2}\right], i=u, d, e .
$$

From (5)-(7) and the $\beta$-equilibrium condition, $\mu_{d}=\mu_{u}+\mu_{e}$, the angle $\theta_{d e}$ that determines the emissivity (4) is obtained

$$
\theta_{d e}^{2} \simeq\left\{\begin{array}{c}
\frac{4}{3 \pi} \alpha_{s} \\
\frac{m_{d}^{2}}{p_{F, e} p_{F, d}}\left[1-\left(\frac{m_{u}}{m_{d}}\right)^{2}\left(\frac{p_{F, d}}{p_{F, u}}\right)-\left(\frac{m_{e}}{m_{d}}\right)^{2}\left(\frac{p_{F, d}}{p_{F, e}}\right)\right]
\end{array}\right.
$$

If interactions and masses are neglected, or the Fermi sea of one species is closed as in the single-flavor CSL phase, it follows that the triangle of momentum conservation in Fig. 4) degenerates to a line or can even not be closed. In that case the matrix element vanishes with the consequence that the direct URCA process does not occur, and also the bulk viscosity is zero. However, in the mixed nuclear-CSL phase there could be important friction and pair-breaking/formation processes, which we have not yet studied in detail. This could be an interesting issue for further investigation due to the large difference in the masses of baryons and deconfined quarks.

\section{MECHANISM FOR DEEP CRUSTAL HEATING}

Superbursts are rare, puzzling phenomena observed as a extremely long (4-14 hours) and energetic ( 10 $\left.{ }^{42} \mathrm{erg}\right)$ type-I X-ray bursts from LMXBs. They take place if the accreted hydrogen and helium at the surface burns in an unstable manner, which is the normal case [44]. As suggested by the authors of Ref. [45], superbursts could originate from accreting strange stars with a tiny crust and a core of three-flavor quark matter in the superconducting color flavor locked (CFL) phase, since it fulfills the constraints on matter properties from their superburst scenario. Of particular importance for the scenario is that in this phase the neutrino emissivity and heat conductivity are suppressed by pairing gaps affecting all quark species [46, 47, 48]. The mechanism underlying the superburst phenomenon is 
unstable thermonuclear burning of carbon in the crust at column depths of about $10^{9} \mathrm{~g} \mathrm{~cm}^{-2}$ [49]. The carbon itself is a remnant of the burning of accreted hydrogen and helium at the surface. The ignition of observed superburst light curves takes place at a depth where the crust reaches temperatures around $6 \times 10^{8} \mathrm{~K}$ and column depths of about $10^{12} \mathrm{~g} \mathrm{~cm}^{-2}$. Such high temperatures in the crust at a certain depth are caused by deep crustal heating $[50,51,52]$. The important ingredients for the strange star model of [45] are a thin baryonic crust of 100 to $400 \mathrm{~m}$ thickness, a sufficient energy release of 1 to $100 \mathrm{MeV}$ per accreted nucleon by conversion into strange matter, a suppression of the fast direct URCA neutrino emissivity to the order of $10^{21} \mathrm{erg} \mathrm{cm}^{-3} \mathrm{~s}^{-1}$, and a thermal conductivity, $\kappa$, of quark matter in the range $10^{19}-10^{22} \mathrm{erg}^{-1} \mathrm{sm}^{-1} \mathrm{~K}^{-1}$.
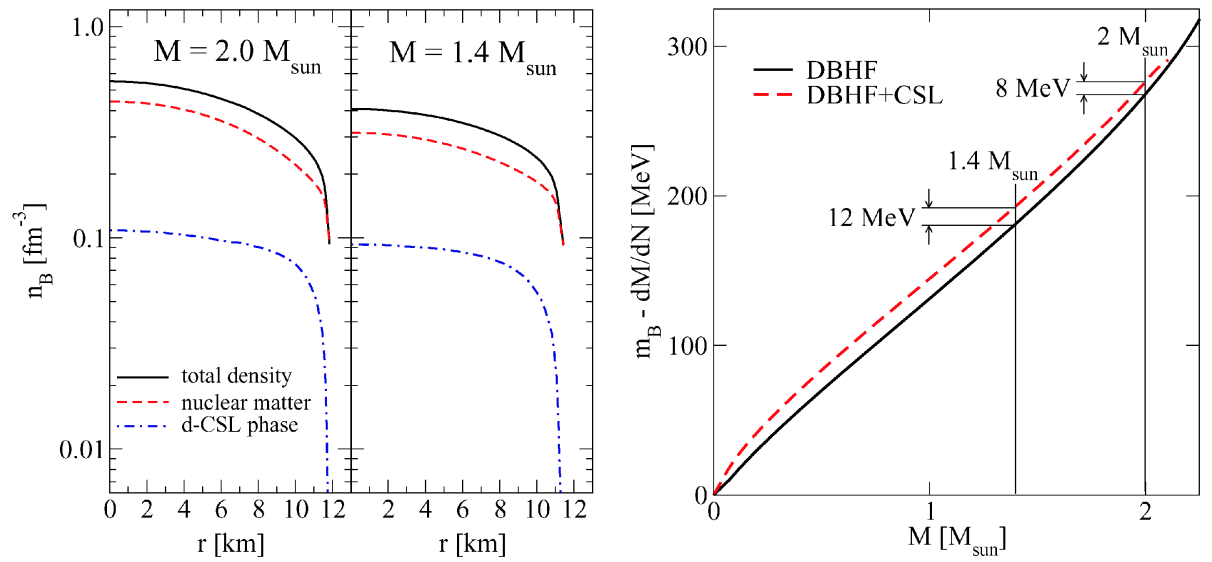

FIGURE 5. Left panel: Density profiles of two stars with masses $1.4 \mathrm{M}_{\odot}$ and $2.0 \mathrm{M}_{\odot}$. Note that the mixed phase of d-CSL quark matter with nuclear matter extends up to the crust-core boundary. Right panel: Energy release per nucleon as a function of the compact star mass. An upper estimate for the energy release from the conversion of DBHF nuclear matter to DBHF-CSL hybrid matter gives $12(8) \mathrm{MeV}$ for a compact star core with mass $1.4(2.0) M_{\odot}$.

One of the main arguments for strange matter is the fact that superconducting phases, like the CFL phase, can suppress fast neutrino emission processes of all quark flavors and are able to fulfill the fusion ignition condition. However, as we have shown above, in the single flavor CSL-phase the fast direct URCA process is not possible at all, whereas slow neutrino cooling processes like bremsstrahlung of electrons and d-quarks occur.

As one can see from Fig. 5 the energy release from the conversion of DBHF nuclear matter to DBHF-CSL hybrid matter gives 12 (8) $\mathrm{MeV}$ for a compact star core with mass $1.4(2.0) M_{\odot}$, which is in the range $1-100 \mathrm{MeV}$ and could, in principle, explain burst ignition at appropriate depths for a suitable value of $\kappa$. Therefore, the compact star does not necessarily need to be made of strange matter, but could be a hybrid star with quark matter in the d-CSL phase and a thin crust. Stejner et al. [44] show that deep crustal heating mechanisms at the crust-core boundary like the conversion of baryonic matter to strange quark matter, which can fulfill the constraints of the superburst scenario do provide a 
consistent explanation of the cooling of soft X-ray transients too. Along the lines of this argument we claim that the d-quark drip effect at the crust core boundary, which leads to a mixture of nuclear matter with single-flavor quark matter in the CSL phase can serve as a deep crustal heating mechanism. Superbursts and the cooling of X-ray transients are not only consistent with quark matter in compact stars but may qualify as a signature!

\section{CONCLUSIONS}

In this contribution we have suggested a new quark-nuclear hybrid EoS for compact star applications that fulfills modern observational constraints from compact stars. Due to isospin asymmetry, down-quarks may "drip out" from nucleons and form a single-flavor color superconducting (CSL) phase that is mixed with nuclear matter already at the crust-core boundary in compact stars. The CSL phase has interesting cooling and transport properties that are in accordance with constraints from the thermal and rotational evolution of compact stars [42]. It remains to be investigated whether this new compact star composition could lead to unambiguous observational consequences [53]. We conjecture that the d-quark drip may serve as an effective deep crustal heating mechanism for the explanation of the puzzling superburst phenomenon and the cooling of X-ray transients.

\section{ACKNOWLEDGEMENTS}

We thank the organizers for bringing together colleagues from different communities and for creating the stimulating atmosphere at the Workshop in Spa, Belgium. D.B. is supported in part by the Polish Ministry of Science and Higher Education, T.K. is grateful for partial support from the Department of Energy, Office of Nuclear Physics, contract no. DE-AC02-06CH11357. The work of F.S. was supported by the Belgian fund for scientific research (FNRS). D.B. and F.S. acknowledge the European Science Foundation for supporting their participation at the Workshop of the Research Networking Programme "The Physics of Compact Stars (CompStar)" in Lądek Zdrój, Poland, where part of this work has been done.

\section{REFERENCES}

1. T. Klähn et al., Phys. Rev. C 74, 035802 (2006).

2. D. Barret, J. F. Olive and M. C. Miller, Mon. Not. Roy. Astron. Soc. 361, 855 (2005).

3. J. E. Trümper, V. Burwitz, F. Haberl and V. E. Zavlin, Nucl. Phys. Proc. Suppl. 132, 560 (2004).

4. F. Özel, Nature 441, 1115 (2006).

5. J. Cottam, F. Paerels and M. Mendez, Nature 420, 51 (2002).

6. C. Fuchs, Prog. Part. Nucl. Phys. 56, 1 (2006).

7. P. Danielewicz, R. Lacey and W. G. Lynch, Science 298, 1592 (2002).

8. M. Alford, D. Blaschke, A. Drago, T. Klähn, G. Pagliara and J. Schaffner-Bielich, Nature 445, E7 (2007). 
9. T. Klähn et al., Phys. Lett. B 654, 170 (2006).

10. D. Blaschke, S. Fredriksson, H. Grigorian, A. M. Öztas and F. Sandin, Phys. Rev. D 72, $065020(2005)$.

11. D. B. Blaschke, D. Gomez Dumm, A. G. Grunfeld, T. Klähn and N. N. Scoccola, Phys. Rev. C 75, 065804 (2007).

12. C. J. Horowitz, E. J. Moniz and J. W. Negele, Phys. Rev. D 31, 1689 (1985).

13. G. Ropke, D. Blaschke and H. Schulz", Phys. Rev. D 34, 3499 (1986).

14. S. Lawley, W. Bentz and A. W. Thomas, J. Phys. G32, 667 (2006).

15. F. de Jong and H. Lenske, Phys. Rev. C 58, 890 (1998).

16. T. Gross-Boelting, C. Fuchs, A. Faessler, Nucl. Phys. A 648, 105 (1999).

17. C. Fuchs, Lect. Notes Phys. 641, 119 (2004).

18. E. N. E. van Dalen, C. Fuchs and A. Faessler, Nucl. Phys. A 744, 227 (2004); Phys. Rev. C 72, 065803 (2005).

19. E. N. E. van Dalen, C. Fuchs and A. Faessler, Phys. Rev. Lett. 95, 022302 (2005).

20. H. Shen, H. Toki, K. Oyamatsu and K. Sumiyoshi, Nucl. Phys. A 637, 435 (1998).

21. S. B. Rüster, V. Werth, M. Buballa, I. A. Shovkovy and D. H. Rischke, Phys. Rev. D 72, $034004(2005)$.

22. H. Abuki and T. Kunihiro, Nucl. Phys. A 768, 118 (2006).

23. H. J. Warringa, D. Boer and J. O. Andersen, Phys. Rev. D 72, 014015 (2005).

24. H. Grigorian, Phys. Part. Nucl. Lett. 4, 382 (2007).

25. M. Buballa, Phys. Rept. 407, 205 (2005).

26. T. Schäfer, Phys. Rev. D 62 (2000) 094007.

27. M. G. Alford, J. A. Bowers, J. M. Cheyne and G. A. Cowan, Phys. Rev. D 67 (2003) 054018.

28. A. Schmitt, Phys. Rev. D $\mathbf{7 1}$ (2005) 054016.

29. D. N. Aguilera, D. Blaschke, M. Buballa, and V. L. Yudichev, Phys. Rev. D 72, 034008 (2005).

30. D. N. Aguilera, D. Blaschke, H. Grigorian and N. N. Scoccola, Phys. Rev. D 74, 114005 (2006).

31. F. Marhauser, D. Nickel, M. Buballa and J. Wambach, Phys. Rev. D 75, 054022 (2007).

32. M. Alford, M. Braby, M. W. Paris and S. Reddy, Astrophys. J. 629, 969 (2005).

33. D. Blaschke and H. Grigorian, Prog. Part. Nucl. Phys. 59, 139 (2007).

34. S. Popov, H. Grigorian, R. Turolla and D. Blaschke, Astron. Astrophys. 448, 327 (2006).

35. S. Popov, H. Grigorian and D. Blaschke, Phys. Rev. C 74, 025803 (2006).

36. H. Grigorian, D. Blaschke and T. Klähn, Neutron Stars and Pulsars, Becker W and Huang H H (eds.), MPE Report 291, 193 (2006).

37. J. Madsen, Phys. Rev. Lett. 85, 10 (1999).

38. A. Drago, G. Pagliara and I. Parenti, Astrophys. J. 678, L117 (2008).

39. N. Andersson, Astrophys. J. 502, 708 (1998).

40. N. Andersson and K. D. Kokkotas, Int. J. Mod. Phys. D 10, 381 (2001).

41. B. A. Sa'd, I. A. Shovkovy and D. H. Rischke, Phys. Rev. D 75, 065016 (2007).

42. D. B. Blaschke and J. Berdermann, AIP Conf. Proc. 964, 290 (2007).

43. N. Iwamoto, Ann. Phys. 141, 1 (1982).

44. M. Stejner and J. Madsen, Astron. Astrophys. 458, 523 (2006).

45. D. Page and A. Cumming, Astrophys. J. 635, L157 (2005).

46. D. Blaschke, T. Klähn and D. N. Voskresensky, Astrophys. J. 533, 406 (2000).

47. D. Page, M. Prakash, J. M. Lattimer and A. Steiner, Phys. Rev. Lett. 85, 2048 (2000).

48. D. Blaschke, H. Grigorian and D. N. Voskresensky, Astron. Astrophys. 368, 561 (2001).

49. A. Cumming and L. Bildsten, Astrophys. J 559, L127 (2001).

50. P. Haensel and J. L. Zdunik, Astron. Astrophys. 227, 431 (1990).

51. G. Ushomirsky and R. E. Rutledge, Mon. Not. Roy. Astron. Soc. 325, 1157 (2001).

52. P. S. Shternin, D. G. Yakovlev, P. Haensel and A. Y. Potekhin, Mon. Not. R. Astron. Soc. 382, L43 (2007).

53. D. Blaschke, F. Sandin, T. Klähn and J. Berdermann, in preparation (2008). 